\title{
War Field Robot with Night Vision Camera
}

\author{
Kavita Mhatre, Nikita Jha, Sonali Dhurway, Jugnum Parimal
}

\begin{abstract}
Considering the current scenario of warfare between India and China, we got inspired by the thought of various ways technology can help our hardworking soldiers by reducing the human loss by using an application that can spy on the enemy, and also for security purpose. This project's main purpose is to deal with difficult situations like where humans cannot go through scenarios like darkness, entering narrow areas and detecting hidden bombs etc. The robot serves as a perfect machine for the defense sector in order to reduce the human life loss and will also help in prevention of illegal activities. The robot is self-powered, with a backtracking facility, in case a situation arises where there is connection loss from the base station. Wireless cameras sends back real-time video and audio inputs that can be seen on a monitor in the base station and action can be taken accordingly.
\end{abstract}

Keywords: Robot, Arduino, Night Vision Wireless Camera, Bluetooth

\section{INTRODUCTION}

$A^{\text {s }}$ we might have seen in the news or read in the newspaper about the ongoing tension among India and China at LAC in Galvan valley and also with Pakistan in J\&K lands. As a youth, we thought of helping our soldiers who are fighting for our safety and well-being by staying on guard for 24hrs not only by risking their lives but also by staying away from their families for months. So, we came up with an idea to help our soldiers by building a war-based spying robot to help them, spy, not only on enemy's movements but also their armoires by providing the intel to our soldiers on war field by gaining not only some time to work on counter strategies but also to know the condition of the territory before entering. As you might have seen in movies like Raazi where the protagonist acts as a spy and provide intel about the enemy which involves risking her life in order to reduce the human loss this robot can be used which not only can reduce the human loss by its ability to go in narrower areas which are

Manuscript received on May 05, 2021.

Revised Manuscript received on May 15, 2021.

Manuscript published on June 30, 2021.

* Corresponding Author

Dr. Kavita Mhatre, Department of Electronics and Communication, Usha Mittal Institute of Technology, Mumbai (M.H), India.

Nikita Jha*, Department of Electronics and Communication, Usha Mittal Institute of Technology, Mumbai (M.H), India. Email: nikita.jha01@gmail.com

Sonali Dhurway, Department of Electronics and Communication, Usha Mittal Institute of Technology, Mumbai (M.H), India. Email: dhurway0603@gmail.com

Jugnum Parimal, Department of Electronics and Communication, Usha Mittal Institute of Technology, Mumbai (M.H), India. Email: pjugnum@gmail.com

(C) The Authors. Published by Blue Eyes Intelligence Engineering and Sciences Publication (BEIESP). This is an open access article under the CC BY-NC-ND license (http://creativecommons.org/licenses/by-nc-nd/4.0/) inaccessible to humans without getting spotted but also its ability to see in the night without getting much attention by using its night vision camera installed on the robot. This technology is used for serial communication with the robot. It is used to share data between 2 devices considering the range betweendevices. The Bluetooth module HC-05 will be connected with the robot and the commands are given to the robot by the android application.

\section{LITERATURE SURVEY}

Beginning with the most basic and important term regarding the project, i.e., the word 'ROBOT'. Literally, this word originates from a salvic word ROBOTA that means labour. Simply saying, a robot is generally a machine that is able to perform certain tasks either automatically or manually or in both ways simultaneously.

Generally, the robots that are used in those battlefields are machines that helps in removing mines and such obstacles in war fields all by itself and sometimes they are also used to spy on enemies. Nowadays, as the domain of technology is getting improvised, robots can be seen being used in military operations as well which are not completely automatic instead, they are controlled remotely. Generally, [4] \& [5] there are three kinds of machines used in military operations namely UGV(Unmanned Ground Vehicle), UAV(Unmanned Ground Vehicle), UUV(Unmanned Underwater Vehicle)

UGV: These are used for land surface purposes and can carry heavy load, move on a rough surfaces and have various sensors and cameras attached to them.

UAV: These are used to carry aerial weapons and flying machines.

UUV: These are actually regarded as submarines or more specifically, machines that can survey underwater.

Next another important machine to be discussed is TISON[1]. This is a heavy EOD(Explosive Ordnance Disposal) robot which is a north American military robot that is capable of lifting up to 100 pounds.

Even though it has a strong gripping mechanism, it has one limitation which is that it cannot function properly at night time. And lastly, S.Naskar[3] tried to explore the field on how a RF controlled robot that can be used in defense and battlefield.

\section{HARDWARE COMPONENTS}

Below are the hardware components used in designing this robot.

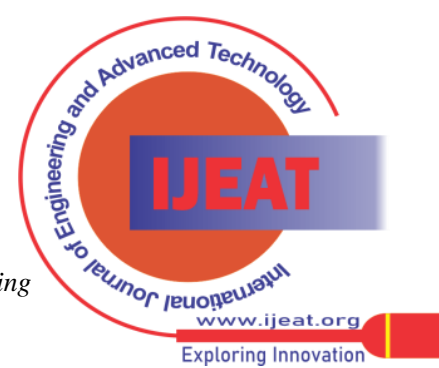




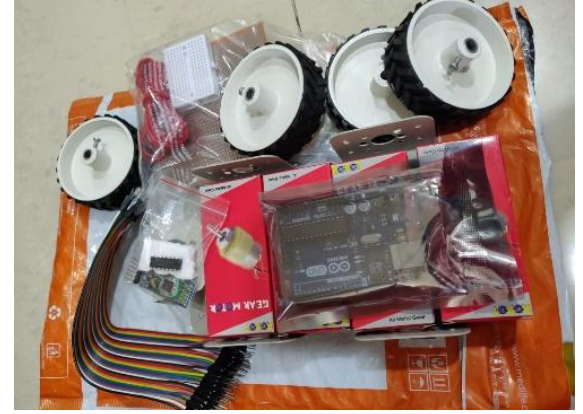

Fig1. All the components used

\section{A. Bluetooth Module HC-05}

This module is capable of adding two-way wireless functionality to the project and also communicates with the two microcontrollers.

This is a simple wireless communication device which is based on Bluetooth protocol. It consists of six pins 1)Key 2)5V3)GND 4)TX 5)RX 6)status. Basically, it uses serial port protocol (SPP). It is designed for wireless serial connection and this can also be used in master or slave configuration. One device is connected to the slave and another is connected to master.

The connection between the devices follows as -

$\mathrm{Rx}$ pin of Arduino is connected to Tx pin of the Bluetooth module while Tx pin of Arduino is connected to the $\mathrm{Rx}$ pin of Bluetooth module. So, in this way, a crossconnection setup is required for the operation of the Bluetooth module and the GND pin of the Bluetooth module is connected to that of the Arduino.

The master device must be connected to the salve in order to have a proper communication. When the pairing is done between two devices, then the device will ask to enter the password. The password will be 0000 or 1234 .

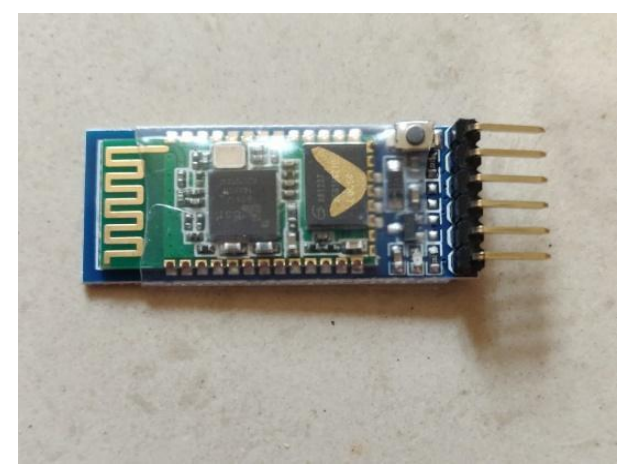

Fig2.HC-05 module

\section{B. Arduino}

As it is an open-source platform which is mostly used inbuilding electronics projects consisting of both a physical programmable circuit board (often referred to as a microcontroller) and a software IDE (Integrated Development Environment) which runs on a computer and is used to write and upload code to the physical board. Unlike its predecessors PCB's, this one doesn't require a separate piece of hardware, i.e., a programmer, In order to load a new code onto the board - one can simply use a USB cable. Also, its IDE uses a simplified version of $\mathrm{C}^{++}$, making it much easier to learn to program.Arduino Uno is also known as a microcontroller board which is based on the ATmega328P processor consisting 14 digital input/output pins (out of which 6 can be used as PWM (to simulate analog outputs, pins marked with $(\sim)$ symbol is used, available on the board), 6 analog inputs(A0-A5), a $16 \mathrm{MHz}$ quartz crystal, a USB connection, a power jack, an ICSP header and lastly a reset button. Also, it comprises everything that is needed to support microcontrollers. In order to get connected to a computer, one has to simply connect the USB cable. It is a blue circuit board, the size of a credit card (also available in other sizes). It's divided into two rows of connectors (the 'headers'), a power connector and a USB connector. An Atmel microcontroller is the brain of the board. It's similar to a really small, very low power 'computer'.

Below are some of its features:

- Photoresistors (sensing light levels)

- On/Off lights, LED's

- Sensors (to sense the environment)

- Ultrasound (proximity range finder)

- Push buttons, touch pads

- Motion detection

- Displays (LCD)

- Thermistors (measuring temperature)

- Actuators (to perform action)

- Motors

- Speakers

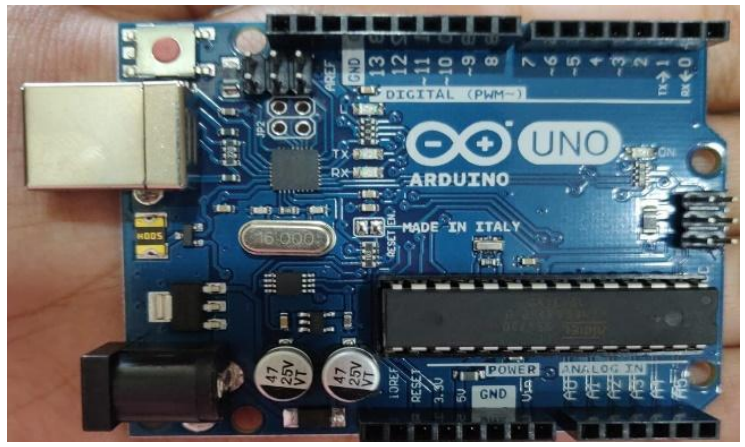

Fig 3. Arduino UNO

\section{L293D IC}

It is a popular 16-pin Motor Driver IC. It is mainly used to drive motors and also it is capable of running two DC motors at the same time.

Motor driver IC is an integrated circuit which is usually used to control motors.L293D has two H-bridge.

L293D Motor Driver IC can control both speed \& spinning direction of two motors. It consists of 16 pins, with 8 pins on each side, for the purpose of controlling the motors. There are 2 I/P pins, 2 O/P pins \& 1 Enable pin for each motor. It provides bidirectional drive currents of up to $600-\mathrm{mA}$ at voltage from $4.5 \mathrm{~V}$ to $36 \mathrm{~V}$.

Published By:

Blue Eyes Intelligence Engineering and Sciences Publication

(C) Copyright: All rights reserved.

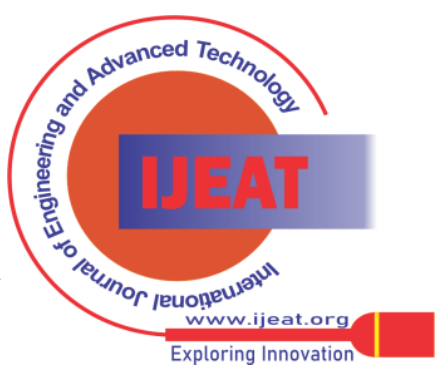




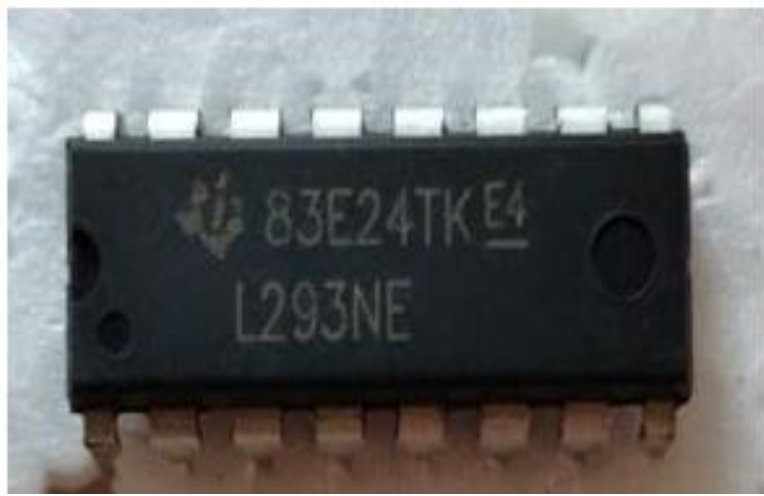

Fig 4. L293 IC

\section{Night Vision Camera}

Human eyes cannot see in the dark light. Hence night vision cameras are used for many purposes specifically in the field of security and surveillance. These devices offer a bit more security than the normal standard cameras. They have the ability to capture objects that are invisible to human eyes either in an image format or recording a video. The specific type used in this project is the night vision wireless camera. It being a wireless device would help us in allowing the device to travel a particular distance without any bound and complete the mission allotted. Below are the features of the night vision wireless camera used:-

- It can automatically detect a movement around that particular area.

- $\quad$ It can allow the transmission of data over 100 meters of range without any obstacles in between.

- $\quad$ A CMOS imaging sensor of size 1/3 inch is installed that detects and converts the information that is used to make an image.

- Next are the video standards along with the resolutions namely PAL(Phase Alternate Line) 628*582/NTSC(National Television Standards Committee) $510 * 492$.

- $\quad$ Illumination is needed to obtain images of a specified desired quality. It provides about min. illumination of 1.5 lux.

- $\quad$ View angle required is 62 Degree

- $\quad$ Camera head used is of 15 gm weightage.

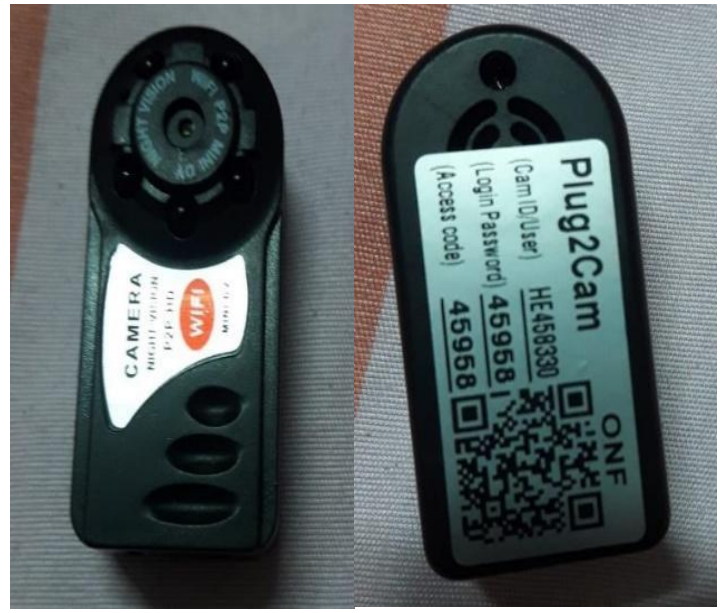

Fig 5. Night Vision Camera

\section{INTERFACING}

\section{A. Interfacing of Bluetooth Module with Arduino}

Bluetooth is a one of the great examples for wireless connectivity. It's applications are in various fields. It consumes almost negligible amount of power. Commonly used Wireless technology are :-

- Wi-Fi

- Bluetooth

This Bluetooth module can communicate in 2

ways, meaning it is full duplex, which can be applied alongwith

microcontrollers. Because it operates Serial Port Protocol (SPP). Using SPP, each connected device is able to send and receive whole data as if there is $\mathrm{Rx}$ and $\mathrm{Tx}$ lines connected between them. 2 Arduino modules, for instance cancommunicate with each other from across rooms, instead from across the desk. The communication is acted upon with the help of USART (Universal Synchronous/Asynchronous Receiver/Transmitter). The HC-05 is capable of operating in 2 modes. One is Data mode and other is AT command (Attention Command used to change default settings of Bluetooth Module). In order to operate in Data Mode of HC05 the enable pin is set to "low", if that pin set as "High" the module operates in AT command mode. For this project we will operate this module in Data Mode.

Table 1

\begin{tabular}{|c|c|}
\hline Arduino UNO & $\mathrm{HC}-05$ \\
\hline $\mathrm{Rx}$ & $\mathrm{Tx}$ \\
\hline $\mathrm{Tx}$ & $\mathrm{Rx}$ \\
\hline $5 \mathrm{~V}$ & $+5 \mathrm{~V}$ \\
\hline GND & GND \\
\hline
\end{tabular}

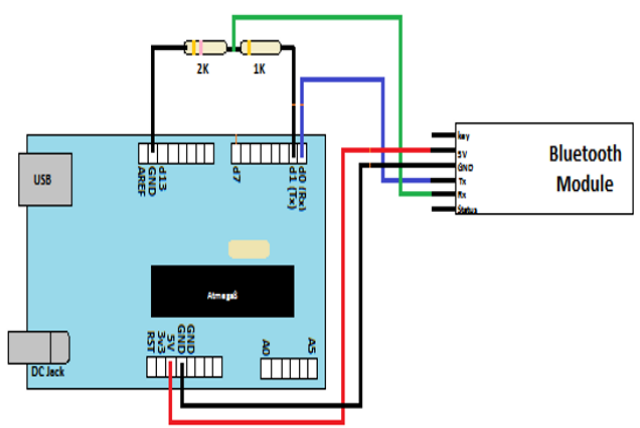

Fig 6. HC-05 Interfacing with Arduino

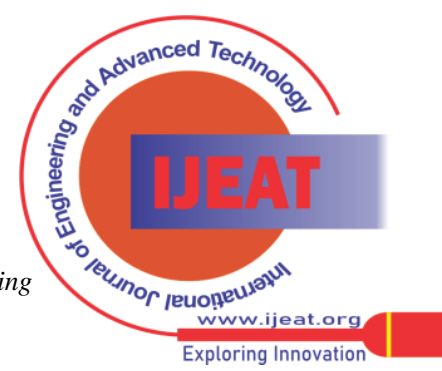




\section{War Field Robot with Night Vision Camera}

\section{B, DC Motor interfacing with Arduino}

The IC LM293D comprises 4-i/p pins, pin 2 and 7 on the left side and Pin 10 and 15 present on the right side of IC respectively. Left input pins of the IC control the rotation of the motor. In accordance with the i/ps that are provided across the

input pins that are Logic 0 and Logic 1 , the motor rotates. Assuming, the motor is connected to the o/p pins 3 and 6 respectively on the left side of the IC. For motors to rotate in clockwise direction, the i/p pins are to be provided with Logic 0 and Logic 1 that is if Pin-2= logic 1 and pin-7 = logic 0 , resulting in the rotation in clockwise direction and vice-versa. Similarly, when Pin-2 $=$ Pin-7=logic 0 , then no rotation is observed and if Pin-2= Pin-7=logic 1 , then also no rotation. In similar way the motor can also be operate across input pins namely pin-15 and pin-10 on the right-hand side.

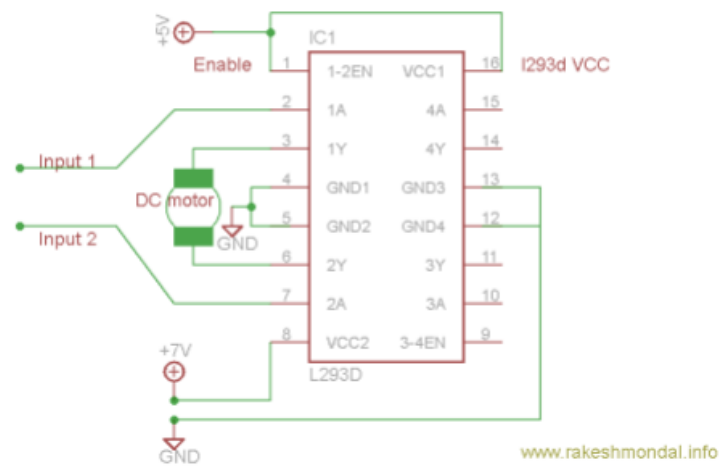

Figure 3.2: DC motors-Arduino interfacing

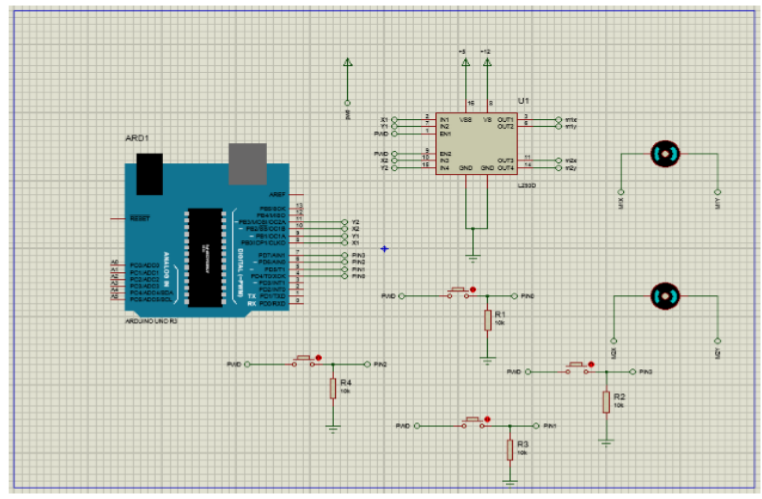

Fig 7. Overall Architecture

\section{FUTURE ENHANCEMENT}

This robot has one limitation which is the range of communication. It cannot be operated over large distances. To increase the range, modules such as Wi-Fi and Zigbee can be used. Zigbee has low power consumption and also has a very long battery life. These modules can also be used to connect this system directly to internet through which we can control the system via any remote areas.

Also, by using DTMF technology we can easily control the robotic machine by using our own mobile phones.

\section{CONCLUSION}

In this paper, a robot is described that uses night vision wireless camera which is operated by an android application. This robot can be further modified using gas sensors as well as bomb diffuse kit. This model of a robot has various applications that include military missions, wireless security and surveillance and search and rescue operation.

\section{REFERENCES}

1. M. Tison, QinetiQ North America, Heavy-lift EOD robot, International Conference, art. 125.

2. K. O. Arras, R. Siegwart, G.Caprari, The autonomous miniature robot Alice: from prototypes to applications, IEEE/RSJ International Conference on Intelligent Robots and Systems, vol.1, 2000, pp. 793798., 2000.

3. S. Das, A. K Seth, A. Nath., S. Naskar, 2011. Application of Radio Frequency Controlled Intelligent Military Robot in Defense. Communication Systems and Network Technologies (CSNT), International Conference, art. 7-50, 2011.

4. Haard Mehta, Jignesh Patoliya , Hitesh Patel, Arduino Controlled War Field Spy Robot using Night Vision Wireless Camera and Android Application, 2015.

5. Md. Baijid Hasan Shorif,Shekh Nuruzzaman3, Ahsanul Hoque, Md. EftekharAlam, Arduino based Battlefield Assistive Robot2017 IEEE Region 10 Humanitarian Technology Conference (R10-HTC), 2017.

\section{AUTHORS PROFILE}

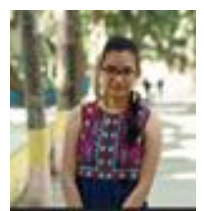

Nikita Jha, Address 1: Flat no-502 Payal Heights plot no-93, sec-19 kharghar, Navi Mumbai 410210Education: B.Tech in Electronics and Communication Designation: Student Date of Birth 1: 31/03/1999

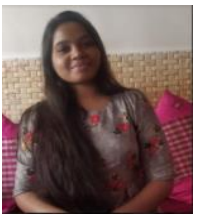

Sonali Dhurway, Address 2: Kunal-2, D/106, Bhau Nagar, Near New Viva College, Bolinj, Virar(W)Education: B.Tech in Electronics and Communication

Designation: Student Date of Birth 2: 06/03/2000

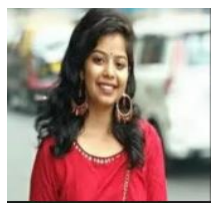

Jugnum Parimal, Address 3: D-13, central Railway Officers Quarter, Sant gadge baba Marge, Off Dadasaheb Phalke Road, Dadar(E), Mumbai400014Education: B.Tech in Electronics and Communication Designation: Student Date of birth 3: 22/11/1998

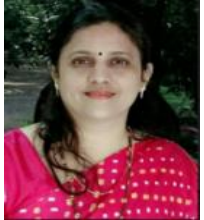

Dr. Kavita Mhatre, Education: Ph.D in Wireless Networks Designation: Associate Professor Experience: 25 years( Teaching + Industry)
Published By:

Blue Eyes Intelligence Engineering and Sciences Publication

(c) Copyright: All rights reserved.

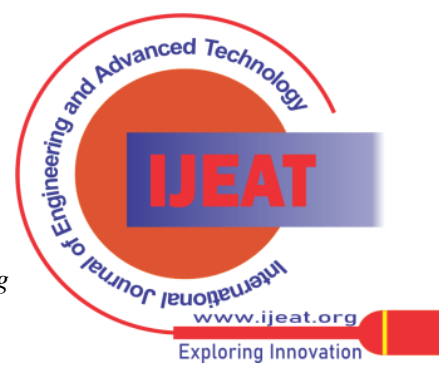

